\title{
ASSOCIAÇÃO ENTRE SINTOMAS DEPRESSIVOS, MOTIVAÇÃO E AUTOESTIMA DE IDOSOS PRATICANTES DE EXERCÍCIOS FÍSICOS'
}

\author{
MS. SIMONE TERESINHA MEURER \\ Mestre em Educação Física pela Universidade Federal de Santa Catarina (UFSC) \\ Doutoranda do Programa de Pós-Graduação em Educação Física da UFSC, Bolsista CAPES \\ (Florianópolis - Santa Catarina - Brasil) \\ E-mail: simonemeurer@yahoo.com.br

\section{MS. LUCÉLIA JUSTINO BORGES} \\ Mestre em Educação Física pela Universidade Federal de Santa Catarina (UFSC) \\ Doutoranda do Programa de Pós-Graduação em Educação Física da UFSC, Bolsista CAPES \\ (Florianópolis - Santa Catarina - Brasil) \\ E-mail: luceliajb@yahoo.com.br

\section{DRA. TÂNIA ROSANE BERTOLDO BENEDETTI} \\ Pós-doutora em Kinesiology and Community Health pela University of Illinois at Urbana e \\ Doutora em Enfermagem pela Universidade Federal de Santa Catarina (UFSC) \\ Docente do Centro de Desportos - CDS/UFSC (Florianópolis - Santa Catarina - Brasil) \\ E-mail: benedetti@cds.ufsc.br
}

\section{DRA. GIOVANA ZARPELLON MAZO}

Doutora em Educação Física pela Universidade do Porto

Docente do Centro de Ciências da Saúde e do Esporte - CEFID,

Universidade do Estado de Santa Catarina - UDESC (Florianópolis - Santa Catarina - Brasil)

E-mail: d2gzm@udesc.br

I. O presente estudo obteve apoio financeiro da Coordenação de Aperfeiçoamento de Pessoal de Nível Superior (CAPES), por meio da concessão de bolsas de estudos em nível de mestrado (desenvolvimento do projeto de pesquisa) e de doutorado (redação do manuscrito). 


\section{RESUMO}

O estudo analisou as associações entre sintomas depressivos, fatores de motivação e autoestima de 83 idosos praticantes de exercícios fisicos. Os instrumentos utilizados foram anamnese, Geriatric Depression Scale (15); escala de avaliação da autoestima de Rosenberg e Inventário de Motivação para prática regular de atividades físicas. Para análise estatística utilizou-se Teste Qui-quadrado ou Teste Exato de Fisher e Correlação de Spearman ( $p<0,05)$. Os resultados evidenciaram relação estatística inversamente proporcional entre autoestima e sintomas depressivos, bem como baixa ocorrência de sintomas depressivos, elevada autoestima e motivação dos idosos. A prática de exercícios físicos parece colaborar para as pontuações positivas encontradas nas variáveis pesquisadas.

PALAVRAS-CHAVE: Idoso; autoimagem; depressão; motivação.

\section{INTRODUÇÃO}

Oaumento expressivo de idosos na população mundial é um acontecimento marcante. No Brasil, em menos de 50 anos o número de idosos aumentou quase $600 \%$ (VERAS, 2007). Segundo dados do último censo, o número de idosos é de 20.590.599, representando I0,8\% da população brasileira (IBGE, 20 I I). Esse contingente populacional requer ações políticas que contemplem a melhora das condições de saúde e de vida dos idosos, uma vez que a maior parte desses possui limitações funcionais e são portadores de doenças crônicas (VERAS, 2007).

Dentre essas doenças, a depressão é uma condição frequente entre as pessoas idosas (DJERNES, 2006; SNOWDON, 2002), e a presença desta pode elevar a probabilidade de incapacidade funcional (RUILOBA, 2007). A literatura tem evidenciado, ainda, que algumas variáveis podem contribuir para o aparecimento dos sintomas depressivos, entre estes, a autoestima vulnerável (HAYES; HARRIS; CARVER, 2004).

Nesse sentido, a autoestima, compreendida como o sentimento, o apreço e a consideração que uma pessoa sente por si própria, é um constructo sociopsicológico de grande importância para a saúde do idoso (ROSENBERG, 1965). Alguns autores sugerem que a autoestima tende a diminuir com o envelhecimento, fato que pode estar relacionado às perdas fisiológicas, de status social ou de ente queridos (FOX, 1997; KRAWCZYNSKI; OLSZEWSKI, 2000).

A prática de exercícios físicos é apontada como uma ação eficaz para manutenção da autoestima positiva de idosos (SPENCE; MCGANNON; POON, 2005; MCAULEY et al., 2005; MEURER; BENEDETTI; MAZO, 2009), bem como na redução de sintomas depressivos (BORGES, BENEDETTI; MAZO, 20I0), sendo inclusive indicado como uma das possibilidades de tratamento para depressão em idosos (FRAZER, CHRISTENSEN, GRIFFITHS, 2005). Todavia, um dos maiores 
desafios é vencer a inatividade física, ainda prevalente entre pessoas deste grupo etário (BRASIL, 20 I2; SIQUEIRA, 2008).

Desta forma, destaca-se que entender os fatores motivacionais pelos quais as pessoas realizam exercícios físicos é uma variável fundamental para intervir sobre o cenário da alta prevalência de inatividade física (BALBINOTTI; BARBOSA, 2006; RYAN; DECY, 2000). Estudos que investigaram a motivação para exercícios físicos, mostram relações desta com a autoestima (WILSON; RODGERS, 2002) e indicaram baixa motivação para a prática de exercícios físicos de pessoas que apresentam sintomas depressivos (KADRI et al., 2009). Ainda assim, observa-se carência de estudos que identifiquem como acontece a relação destas três variáveis em idosos fisicamente ativos. Acredita-se que, com este conhecimento, pode-se ampliar e melhorar as propostas de programas dessa natureza, no sentido de contribuir para a melhora da autoestima, diminuição de sintomas depressivos e aumento da motivação para prática de exercícios físicos.

Desta forma, objetivou-se neste estudo analisar as associações entre sintomas depressivos, fatores de motivação e autoestima de idosos praticantes de exercícios físicos.

\section{MATERIAIS E MÉTODOS}

O delineamento de pesquisa empregado foi transversal.

A população foi constituída por idosos (aproximadamente 250) cadastrados no programa de exercício físico denominado Floripa Ativa - Fase "B", que é realizado nos Centros de Saúde (CS) de Florianópolis, desde 2006. Esse programa é destinado aos idosos usuários dos CS, que tem por objetivo a prevenção secundária e terciária, sendo uma das ações de atenção ao idoso da Secretaria Municipal de Saúde de Florianópolis. Maiores detalhes desse programa foram descritos previamente em outros estudos (MEURER; BENEDETTI; MAZO, 20 I I; BORGES; BENEDETTI; MAZO, 2010 ; BORGES; BENEDETTI; MAZO, 2007).

A amostra foi do tipo intencional, sendo utilizados os seguintes critérios para inclusão: ter idade igual ou superior a 60 anos; ter respondido as escalas de rastreamento para depressão, avaliação da autoestima e o inventário de motivação para a prática regular de atividades físicas. Diante desses critérios, a amostra foi constituída por 83 idosos (67 mulheres e 16 homens), com idade entre 60 e 88 anos.

Em relação aos instrumentos, para esse estudo foram utilizados: ficha contendo informações demográficas e socioeconômicas (idade, sexo, estado civil, renda e escolaridade); Geriatric Depression Scale (GDS) - versão abreviada com I 5 perguntas (YESAVAGE et al., 2002); Escala de avaliação da autoestima de Rosenberg (I965) 
(RSES), cuja pontuação varia de 10 a 40 pontos, e o Inventário de Motivação para a Prática Regular de Atividades Físicas (IMPRAF), que é válido para pessoas de 13 a 83 anos e compreende a motivação como os fatores que levam a pessoa a realizar atividades físicas regulares (BALBINOTTI; BARBOSA, 2006).

Para a GDS, utilizou-se a classificação proposta pelo estudo de validação da escala para o Brasil, sendo o indivíduo classificado com suspeita à depressão quando o valor total era maior que cinco pontos (ALMEIDA; ALMEIDA, 1999). Para o IMPRAF, utilizaram-se as tabelas normativas fornecidas no manual de aplicação do instrumento, sendo que os escores brutos são transformados em percentis, o que permite comparar o desempenho do avaliado em seu grupo, de acordo com sexo e idade. As pontuações acima do percentil 50 foram categorizadas como motivação alta.

A coleta de dados foi realizada no período entre julho e dezembro de 2008. A equipe de coleta de dados foi composta pelas pesquisadoras do estudo, auxiliadas por acadêmicos do curso de Educação Física da Universidade Federal de Santa Catarina (UFSC) e Universidade do Estado de Santa Catarina (UDESC). Toda a equipe de coleta de dados foi previamente treinada para a aplicação dos instrumentos.

Esta pesquisa encontra-se aprovada pelo Comitê de Ética em Pesquisa envolvendo Seres Humanos da UFSC. Os indivíduos que aceitaram participar da pesquisa assinaram o Termo de Consentimento Livre e Esclarecido (TCLE).

Os dados foram organizados no Exce ${ }^{\circledR}$ e analisados por meio do pacote estatístico SPSS ${ }^{\circledR}$ para Windows, versão 1 I.5.

A estatística descritiva (média, desvio padrão, frequência absoluta e percentual) foi realizada para a elaboração do perfil demográfico e sócio-econômico da amostra e para a classificação percentual da GDS, da Escala de autoestima e do IMPRAF.

Após verificar que os dados não apresentaram distribuição normal, foi utilizado o Teste Qui-quadrado ou Teste Exato de Fisher para avaliar associação entre as categorias da GDS e dos fatores de motivação. A Correlação de Spearman foi utilizada para verificar a relação entre a pontuação da GDS com a motivação; e entre a autoestima e a pontuação da GDS. Foi adotado para todas as análises o nível de significância de 5\%.

\section{RESULTADOS}

A média de idade dos idosos foi de 68,2 (SD=6,2), e em sua maioria, eram mulheres, na faixa etária de 60 a 69 anos, casadas, com quatro a sete anos de estudo e renda familiar até dois salários (TABELA I). 

Centros de Saúde. Florianópolis, Santa Catarina, Brasil. 2008

\begin{tabular}{lcc}
\hline & Frequência & $\%$ \\
\hline Sexo & & \\
Masculino & 16 & 19,3 \\
$\quad$ Feminino & 67 & 80,7 \\
Idade (anos) & 54 & 65,1 \\
$\quad 60-69$ & 25 & 30,1 \\
$60-79$ & 04 & 4,8 \\
80 ou mais & & \\
Escolaridade (anos de estudo formal)* & 5,2 \\
Nenhum & 04 & 19,5 \\
I - 3 & 15 & 42,9 \\
$4-7$ & 33 & 32,5 \\
8 anos ou mais & 25 & \\
Estado civil & & 50,6 \\
Casado & 42 & 38,6 \\
Viúvo & 32 & 10,8 \\
Separado; Solteiro; Divorciado & 09 & \\
Renda familiar (salários)* & & 42,9 \\
Até 2 & 33 & 37,7 \\
3 a 4 & 29 & 19,5 \\
5 ou mais & 15 &
\end{tabular}

* $n=77$ (06 casos não declarados)

Em relação à motivação dos idosos para a participação no programa de exercícios físicos, identificou-se que a maioria tem "motivação alta" para os fatores investigados, exceto para a estética. As maiores médias foram observadas para os fatores saúde (média =37,25; DP=4,23), sociabilidade (média =36,02; DP=5,69) e prazer ( $m e ́ d i a=34,43 ; \mathrm{DP}=6,78$ ). Em relação aos sintomas depressivos, a maioria dos idosos $(89,2 \%)$ apresentou pontuação dentro do normal, sendo que a mediana dos escores foi dois pontos.

$\mathrm{Na}$ associação entre sintomas depressivos e fatores motivacionais não foi observado significância estatística para nenhum dos fatores motivacionais (TABELA 2). 
Tabela 2. Associação entre sintomas depressivos e fatores motivacionais em idosos praticantes de exercício físico em Centros de Saúde. Florianópolis, Santa Catarina, Brasil. 2008.

Fatores de Motivação

Sintomas Depressivos

Ausência Presença

p valor

Controle de Estresse

$\begin{array}{llll}\text { Baixa } & 26 & 03 & 0,6 \mid 4^{+} \\ \text {Alta } & 48 & 06 & \end{array}$

Saúde

Baixa

19

02

Alta

55

07

$0,592^{+}$

Sociabilidade

$\begin{array}{llc}\text { Baixa } & 14 & - \\ \text { Alta } & 60 & 09\end{array}$

Competitividade

$\begin{array}{llll}\text { Baixa } & 26 & 01 & 0,140^{+} \\ \text {Alta } & 48 & 08 & \end{array}$

Estética

$\begin{array}{llll}\text { Baixa } & 45 & 05 & 0,514^{+} \\ \text {Alta } & 29 & 04 & \end{array}$

Prazer

Baixa 25

Alta 49 04 05

t Teste Exato de Fisher.

Verifica-se que a maioria dos idosos tem autoestima elevada (média = 36; $\mathrm{DP}=4,25)$. Ao relacionar a pontuação dos sintomas depressivos com a autoestima, observou-se uma relação significante e inversa ( $r h o=-0,423 ; p<0,00$ I), porém o mesmo não foi constatado entre a pontuação dos fatores motivacionais (TABELA 3). 
Tabela 3. Associação entre sintomas depressivos, fatores de motivação e autoestima em idosos praticantes de exercício físico em Centros de Saúde. Florianópolis, Santa Catarina, Brasil. 2008

\begin{tabular}{|c|c|c|}
\hline \multirow{2}{*}{ Fatores de Motivação } & Sintomas depressivos & \multirow{2}{*}{$p$ valor } \\
\hline & $\mathrm{R}$ & \\
\hline Controle de Estresse & 0,111 & $0,317^{+}$ \\
\hline Saúde & $-0,|2|$ & $0,274^{+}$ \\
\hline Sociabilidade & $-0,065$ & $0,557^{+}$ \\
\hline Competitividade & 0,118 & $0,288^{+}$ \\
\hline Estética & 0,043 & $0,699^{+}$ \\
\hline Prazer & $-0,077$ & $0,491^{+}$ \\
\hline Autoestima & $-0,423$ & $<0,00 \mathrm{I}^{+}$ \\
\hline
\end{tabular}

${ }^{\dagger}$ Correlação de Spearman.

\section{DISCUSSÃO}

A presente pesquisa investigou a relação entre sintomas depressivos, autoestima e fatores de motivação em idosos praticantes de exercício físico, com participação mínima de seis meses e máxima de dois anos no Programa Floripa Ativa - Fase "B".

Os resultados obtidos demonstram que não houve associação estatística significante entre sintomas depressivos e fatores de motivação. Diferentemente desses resultados, estudo realizado por Kadri et al. (2009), que buscou identificar o quanto motivados estavam as pessoas com sintomas depressivos para a participação regular em um programa de caminhada nórdica, verificou associação entre a presença de sintomas depressivos e baixa motivação para a prática de exercícios físicos.

Outros estudos têm evidenciado a presença de sintomas depressivos como uma importante barreira para a aderência de idosos a exercícios físicos. Forkan, Pumper e Smyth (2006) investigaram os fatores que interferiram sobre a aderência de 556 idosos a um programa de exercícios físicos e identificaram a presença de sintomas depressivos como uma das oito principais barreiras. Já Williams e Lord (1995), ao investigarem a aderência de 102 mulheres, com idade entre 60 e 85 anos, a um programa de exercícios físicos, confirmaram que a baixa pontuação nos fatores relacionados à depressão foi um preditor que auxiliou na identificação daquelas idosas que completaram o programa de 12 meses.

Desta forma, os resultados da presente investigação parecem diferir do que vem sendo mostrado na literatura. Para tanto, deve-se considerar as características da amostra estudada, que parece diferenciar-se de idosos que nunca participaram de 
programas dessa natureza. Ou seja, ao ingressar no programa, os idosos poderiam estar mais motivados para a prática de atividade física e, consequentemente, apresentarem autoestima mais elevada, mais ânimo e disposição para participarem do grupo. Nesse sentido, parece que a não associação estatística identificada pode ser explicada pelo baixo número de pessoas com sintomas depressivos e alto número de pessoas com alta motivação. Vale ressaltar que a prática regular de exercício físico contribui para a baixa incidência de sintomas depressivos (BABYAK et al., 2000) e redução dos sintomas depressivos (BORGES; BENEDETTI; MAZO, 20 I0). Além disso, sentir-se menos deprimido parece ser um fator diretamente relacionado com a motivação elevada para continuar praticando exercícios físicos (KRITZ-SILVERSTEIN; BARRETT-CONNOR; CORBEAU, 200I).

Quando os dados foram tratados de forma contínua, também não foi observada associação estatisticamente significante entre os sintomas depressivos e os fatores motivacionais. Para tal compreensão, utilizar-se-á a Teoria da Autodeterminação, que aborda a motivação como um continuum, podendo variar desde a sua forma mais autônoma até aquela controlada por aspectos externos, incluindo a motivação intrínseca, extrínseca e amotivação (RYAN; DECl, 2000).

Mesmo não sendo detectada associação significante, observou-se tendência dos fatores de motivação com regulação intrínseca (saúde, sociabilidade, prazer) se associarem negativamente com a pontuação para sintomas depressivos, enquanto os fatores de regulação extrínseca (competitividade, controle de estresse, estética) tiveram tendência de se associarem positivamente com os sintomas depressivos. Tais fatores podem ser compreendidos considerando que a amostra dessa investigação foi composta, em sua maioria, por idosos jovens (60 a 69 anos) e que as características biopsicossociais diferenciam dos grupos etários mais velhos. Especificamente a respeito dos fatores de motivação com regulação extrínseca, os fatores elencados parecem estar mais presentes em idosos jovens.

A tendência de associação entre os fatores de motivação e os sintomas depressivos é explicada pela satisfação das necessidades psicológicas básicas, uma vez que o desenvolvimento da motivação intrínseca somente é possível quando houver a satisfação das necessidades de autonomia, competência e relacionamento (RYAN; DECl, 2000). Desta forma, os idosos participantes da presente pesquisa apresentaram maior pontuação para os fatores com regulação intrínseca e tiveram baixas pontuações para os sintomas depressivos. Esse resultado corrobora a pesquisa de Wei et al. (2005), que também identificou associação negativa entre a satisfação das necessidades psicológicas básicas e a presença de sintomas depressivos.

Ao analisar a associação entre sintomas depressivos e autoestima, observou-se que estas se relacionam inversamente, ou seja, quanto mais elevada a autoestima, 
menor a pontuação para sintomas depressivos. Em consonância com este resultado, Meyler, Stimpson e Peek (2006), em pesquisa realizada com 3050 idosos e utilizando a escala de autoestima de Rosenberg, também identificaram associação inversa e moderada entre a autoestima e os sintomas depressivos, sugerindo que a autoestima interfere sobre diferentes aspectos da saúde mental de idosos.

Nesse sentido, a relação entre a autoestima e os sintomas depressivos é explicada pelo modelo teórico proposto por Roberts e Monroe (1994). Os autores sugerem que a vulnerabilidade da autoestima potencializa outras dificuldades, como por exemplo, regular as emoções, os conflitos interpessoais e a regulação do estresse. Estes, por sua vez, podem perpetuar a vulnerabilidade da autoestima e finalmente resultar em depressão.

Considerando a baixa pontuação para sintomas depressivos e a elevada pontuação para a autoestima dos idosos participantes do presente estudo, aponta-se o efeito positivo da prática de exercícios físicos, o que está em consonância com a literatura. Mcauley et al. (2000), em estudo realizado com 174 idosos de um programa de caminhadas ou resistência muscular, evidenciou que um ano ou mais de prática de exercícios físicos colabora para melhorias da autoestima, atribuído às melhorias da aptidão física, diminuição da gordura corporal e aumento da autoeficácia. Em estudo de revisão, Spence, McGannon e Poon (2005) analisaram I I 3 estudos que versavam sobre a relação entre exercícios físicos e autoestima, e concluíram que essa prática influencia na percepção da autoeficácia, da competência e aceitação física, interferindo positivamente sobre a autoestima global.

A presente pesquisa investigou alguns aspectos que garantem a sua singularidade, uma vez que o estudo da relação entre os sintomas depressivos, fatores de motivação e autoestima ainda é incipiente na literatura. E, especificamente em idosos praticantes de exercícios físicos, o estudo da relação entre essas variáveis parece ser pioneiro. Todavia, deve-se considerar algumas limitações. A presente investigação não considerou algumas variáveis que poderiam contribuir como fator de confusão ou para o melhor entendimento das relações, tais como: controle de utilização de medicamentos antidepressivos e assiduidade dos indivíduos no programa de exercício físico. Além disso, o número reduzido da amostra, e, consequentemente, o baixo número de indivíduos que apresentaram suspeita à depressão, baixa motivação e baixa autoestima, podem ter contribuído para a não associação entre algumas variáveis. Outrossim, a carência de estudos com características semelhantes à presente pesquisa pode ter dificultado a discussão dos resultados.

Considerando as limitações, salienta-se que os resultados do presente estudo devem ser extrapolados com cautela e somente para populações com características semelhantes, uma vez que a amostra foi intencional e não representativa. 
Os resultados evidenciaram que os idosos praticantes de exercícios físicos apresentaram motivação alta para a maioria dos fatores investigados, elevada autoestima e baixos escores de sintomas depressivos (pontuação dentro do normal). Foi detectada relação estatística inversamente proporcional entre a pontuação da autoestima e os sintomas depressivos. Contudo, não foi identificada associação entre os fatores de motivação e os sintomas depressivos.

Diante dos resultados, evidencia-se que a prática de exercício físico parece ser um fator importante para a baixa ocorrência de sintomas depressivos, elevada autoestima e motivação dos idosos.

Sugere-se a realização de estudos longitudinais que possam avaliar a associação dessas variáveis e buscar compreender as relações de causalidade e efeito entre as mesmas, bem como, controlar as variáveis mencionadas como fatores limitantes do presente estudo.

Association between depressive symptoms, motivational factors and self-esteem in elderly engaged in physical exercises

ABSTRACT: The study examined the associations between depressive symptoms, motivational factors and self-esteem in 83 elderly engaged in physical exercises. The instruments used were: anamnese, geriatric depression scale (15), Rosenberg self-esteem scale and motivation inventory for regular practice of physical activity. The associations were verified by the Chi-square or Fisher's test and the Spearman correlation, adopting a significance level of 5\%. It was statistically observed that there is an inversely proportional relationship between self-esteem and depressive symptoms, as well as low occurrence of depressive symptoms, high self-esteem and motivation of the elderly. The practice of physical exercises seems to contribute to the positive scores found in the variables studied.

KEYWORDS: Elderly; self concept; depression; motivation.

Asociación entre síntomas depresivos, motivación y autoestima de ancianos practicantes de ejercicios físicos

RESUMEN: El estudio examinó la asociación entre síntomas depresivos, autoestima y factores de motivación de 83 ancianos practicantes de ejercicios físicos. Los instrumentos fueron la entrevista, escala de depresión geriátrica (15), escala de evaluación de la autoestima de Rosenberg y inventário de motivación para la práctica regular de actividades físicas. Para el análisis estadístico se utilizó chi-cuadrado o la prueba exacta de Fisher y la correlación de Spearman $(p<0,05)$. Fue demostrada la relación estadística inversamente proporcional entre la autoestima y los síntomas depresivos, así como la baja incidencia de síntomas depresivos, alta autoestima y motivación de las personas mayores. La práctica de ejercicios físicos parece contribuir a los puntajes positivos encontrados en las variables estudiadas.

PALABRAS CLAVE: Anciano; autoimagen; depresión; motivación. 


\section{REFERÊNCIAS}

ALMEIDA, O. P.; ALMEIDA, S. A. Confiabilidade da versão brasileira da Escala de Depressão Geriátrica (GDS) versão reduzida. Arquivos de Neuro-Psiquiatria, v. 57, n. 2-B, p. 421-426, 1999.

BABYAK, $M$. et al. Exercise treatment for major depression: maintenance of therapeutic benefit at 10 months. Psychosomatic Medicine, New York, v. 62, n. 5, p. 633-638, 2000.

BALDINOTTI, M. A. A.; BARBOSA, M. L. L. Proposta inicial de manual técnico de aplicação do inventário de motivação à prática regular de atividades físicas (IMPRAF - 54). Porto Alegre: Universidade Federal do Rio Grande do Sul, 2006.

BORGES, L. J.; BENEDETTI, T. R. B; MAZO, G. Z. Influencia del ejercicio físico en los síntomas depresivos y en la aptitud funcional de ancianos en el sur de Brasil. Revista Española de Geriatría y Gerontología, Madrid, v. 45, n. 2, p. 72-78, 2010.

BORGES, L. J.; BENEDETTI, T. R. B; MAZO, G. Z. Rastreamento cognitivo e sintomas depressivos em idosos iniciantes em programa de exercício físico. Jornal Brasileiro de Psiquiatria, Rio de Janeiro, v. 56, n. 4, p. 273-279, 2007.

BRASIL. Ministério da Saúde. Vigitel Brasil 201 l : vigilância de fatores de risco e proteção para doenças crônicas por inquérito telefônico. Brasília, 2012.

DJERNES, J. K. Prevalence and predictors of depression in populations of elederly: a review. Acta Psychiatrica Scandinavica, Copenhagen, v. II3, n. 5, p. 372-387, maio 2006.

FORKAN, R.; PUMPER, B. SMYTH, N. Exercise Adherence Following Physical Therapy Intervention in Older Adults with impaired balance. Physical Therapy, Alexandria, v. 86, n.3, p. $401-410,2006$.

FOX, K.R. The physical self and processes in self-esteem development. In: KENNETH, R. F. (Ed.). The physical self - from motivation to well- being. Champaign.: Human Kinetics, 1997. p. || |-139.

FRAZER, C. J; CHRISTENSEN, H; GRIFFITHS, K. M. Effectiveness of treatments for depression in older people. Medical Journal of Australia, Sydney, v. I 82, n. 12, p. 627-632, 2005.

HAYES, A. M.; HARRIS, M. S; CARVER, C. S. Predictors of self-esteem variability. Cognitive Therapy and Research, New York, v. 28, n. 3, p. 369-385, 2004.

INSTITUTO BRASILEIRO DE GEOGRAFIA E ESTATISTICA (IBGE). Sinopse do censo demográfico 20 10. Disponível em: <http://www.ibge.gov.br/home/estatistica/populacao/ censo2010/tabelas_pdf/Brasil_tab_I_I2.pdf >. Acesso em: I 4 set. 201 I.

KADRI, S. et al. Physical activity of depressed patients and their motivation to exercise: nordic walking in family practice. International Journal of Rehabilitation Research, Rheinstetten, v. 32, n. 2, p. 132-138, 2009. 
KRAWCZYNSKI, M.; OLSZEWSKI, H. Psychological well-being associated with a physical activity programme for persons over 60 years old. Psychology of Sport and Exercise, Amsterdam, v. I, n. I, p. 57-63, 2000.

KRITZ-SILVERSTEIN, D.; BARRETT-CONNOR, E.; CORBEAU, C. Cross-sectional and prospective study of exercise and depressed mood in the elderly. American Journal of Epidemiology, Baltimore, v. I53, n. 6, p. 596-603, 2001.

LEITE, V. M. M. et al. Depressão e envelhecimento: estudo nos participantes do Programa Universidade Aberta à Terceira Idade. Revista Brasileira Saúde Materno Infantil, Recife, v. 6, n. I, p. 3 I-38, 2006.

MCAULEY, E. et al. Physical activity, self-efficacy, and self-esteem: longitudinal relationships in older adults. Journal of Gerontology: psychological sciences, Cary, v. 60B, n. 5, p. 268-275, 2005.

MEURER, S. T; BENEDETTI, T. R. B; MAZO, G. Z. Aspectos da autoimagem e autoestima de idosos ativos. Motriz, Rio Claro, v. I5, n.4, p.788-796, 2009.

Teoria da autodeterminação: compreensão dos fatores motivacionais e autoestima de idosos praticantes de exercícios físicos. Revista Brasileira de Atividade Física e Saúde, Londrina, v. I6, n. I, 201 I.

MEYLER, D; STIMPSON, J. P.; PEEK, M.K. Acculturation and self-esteem among older mexican americans. Aging \& Mental Health, Abingdon, v. I0, n. 2, p. I82- 186, 2006.

OLIVEIRA, D. A. A. P; GOMES L.; OLIVEIRA, R. F. Prevalência de depressão em idosos que freqüentam centros de convivência. Revista de Saúde Pública, São Paulo, v. 40, n. 4, p.734-736, 2006.

PARADELA, E. M. P.; LOURENÇO, R. A.; VERAS, R. P. Validação da escala de depressão geriátrica em um ambulatório geral. Revista de Saúde Pública, São Paulo, v. 39, n. 6, p. 918923, 2005.

ROBERTS, J. E.; MONROE, S. M. A multidimensional model of self-esteem in depression. Clinical Psychology Review, Tarrytown, v. 14, n. 3, p. 161-181, 1994.

ROSENBERG, M. Society and the adolescent self image. Princeton: Princeton University, 1965.

RUILOBA, J. V. Current situation of long-term treatment of depression. Actas Españolas de Psiquiatria, Madrid, v. 35, n. 5, p. 285-299, 2007.

RYAN, R. M.; DECI, E. L. Intrinsic and extrinsic motivations: classic definitions and new directions. Contemporary Educational Psychology, San Diego, v. 25, n. I, p. 54-67, 2000.

SIQUEIRA, F. V. et al. Atividade física em adultos e idosos residentes em áreas de abrangência de unidades básicas de saúde de municípios das regiões Sul e Nordeste do Brasil. Caderno de Saúde Pública, Rio de Janeiro, v. 24, n. I, p. 39-54, 2008. 
SNOWDON, J. How high is the prevalence of depression in old age. Revista Brasileira de Psiquiatria, São Paulo, v. 24, Suppl. I, p. S42-S47, 2002.

SPENCE, J. C.; MCGANNON, K. R; POON, P. The effect of exercise on global self-esteem: a quantitative review. Journal of Sport \& Exercise Psychology, Florida, v. 27, n. 3, p. 3 I I -334, 2005.

VERAS, R. Envelhecimento populacional e as informações de saúde do PNAD: demandas e desafios contemporâneos. Introdução. Caderno de Saúde Pública, Rio de Janeiro, v. 23, n. I0, p. 2463-2466, 2007.

WEI, M. et al. Adult attachment, shame, depression, and loneliness: the mediation role of basic psychological needs satisfaction. Journal of Counseling Psychology, Washington, v. 52, n. 4, p. 591-60I, 2005.

WILLIAMS, P.; LORD, S. R. Predictors of adherence to a structured exercise program for older women. Psychology and Aging, Washington, v. I0, n. 4, p. 617-624, 1995.

WILSON, P. M.; RODGERS, W. M. The relationships between exercise motives and physical self-esteem in female exercise participants: an application of self-determination theory. Journal of Applied Biobehavioral Research, Columbia, v.7, n. I, p.30-43, 2002.

YESAVAGE, J. A. et al. Escala de depressão geriátrica (Abreviada de Yesavage, 1983). In: FREITAS, E. V. (Org.). Tratado de geriatria e gerontologia. Rio de Janeiro: Guanabara Koogan, 2002. p. $\mid 154$.

Recebido: 3 dez. 2010 Aprovado: 21 out. 201 I

Endereço para correspondência Simone Teresinha Meurer Núcleo de Cineantropometria e Desempenho Humano, Campus Universitário, Trindade. CEP: 88040-900. Florianópolis. Santa Catarina. 